\title{
Typhoid Intestinal Perforation in Children: Still a Persistent Problem in a Developing Country
}

\author{
Chukwubuike Kevin Emeka ${ }^{a,{ }^{*}}$ \\ ${ }^{a}$ Pediatric Surgery Unit, Department of Surgery, Enugu State University Teaching Hospital, Enugu, Nigeria.
}

\section{Abstract}

Background: Typhoid intestinal perforation is serious complication of typhoid fever and is associated with significant morbidity and mortality in developing countries. The aim of this study was to determine the incidence, pattern and outcome of treatment of typhoid intestinal perforation (TIP) in children in a teaching hospital in Enugu, Nigeria.

Methods: This was a retrospective study of patients aged 15 years and below operated on for TIP in the pediatric surgery unit of Enugu State University Teaching Hospital (ESUTH) Enugu, Nigeria. Medical records of pediatric patients who had laparotomy for TIP over a 10-year period were evaluated.

Results: There were 200 cases of TIP and this accounted for $35.7 \%$ of all pediatric surgeries performed during the period of the study. There were 150 (75\%) males and 50 (25\%) females which correspond to a male female ratio of 3:1. The ages of the patients ranged from 6 to 15 years with a median of 11 years. One hundred percent of the patients had fever and abdominal pain. Anemia, hypokalemia and a single ileal perforation were recorded in most of the patients. Primary closure of the perforation was the most common performed surgical procedure. Surgical site infection, burst abdomen and residual intra-peritoneal abscess accounted for most of the post-operative complications. Mortality was $8.5 \%$.

Conclusions: TIP is still a problem in developing countries due to absence of potable drinking water, poor hygiene and improper waste disposal.

Keywords: Developing country; intestinal perforation; typhoid; persistent
\end{abstract}

\section{INTRODUCTION}

Typhoid fever is a public health problem that is seen mostly in developing countries due to lack of potable drinking water, poor hygiene and improper waste disposal ${ }^{[1]}$. It is multisystem infection caused by the bacteria, Salmonella enterica serovar typhi and Salmonella enterica serovar paratyphi A and B which are transmitted through feco-oral route by ingestion of contaminated food and/or water ${ }^{[2]}$. Among the complications of typhoid fever of surgical importance, typhoid intestinal perforation (TIP) is the most dreaded ${ }^{[3]}$. However, ty-

*Corresponding author: Chukwubuike Kevin Emeka

Mailing address: Department of Surgery, Enugu State University

Teaching Hospital, Enugu, Nigeria.

E-mail: chukwubuikeonline@yahoo.com

Received: 30 March 2020 Accepted: 28 Apirl 2020 phoid intestinal bleeding has been reported as the most common gastrointestinal complication of typhoid fever $[4,5]$. There are wide differences between the incidence of typhoid intestinal perforation in developing and developed countries ${ }^{[6,7]}$. Some researchers have suggested that the higher virulence of Salmonella organisms seen in Sub-Saharan Africa may account for the higher propensity to perforation in Africans ${ }^{[8]}$. A systemic literature review showed that Nigeria has the highest rate of typhoid intestinal perforation in developing countries ${ }^{[9]}$. However, the health altitude of Nigerians who seek unorthod ox treatment and poor disease database make the exact incidence of typhoid intestinal perforation unknown ${ }^{[10]}$. The aim of this study was to determine the incidence, pattern and outcome of treatment of typhoid intestinal perforation in children in a teaching hospital in Enugu, Nigeria.

METHODS 
This was a retrospective study of children aged 15 years and below who had laparotomy for TIP between September 2008 and August 2018 at the pediatric surgery unit of Enugu State University Teaching Hospital (ESUTH) Enugu, Nigeria. The diagnosis of typhoid intestinal perforation was made based on clinical features, investigations, intra-operative findings and histopathological confirmation. On presentation at the children emergency room, all the patients were resuscitated and stabilized using intravenous fluids and antibiotics. A nasogastric tube and urethral catheter were passed to decompress the upper gastrointestinal tract and monitor urine output respectively. Blood was transfused when the hemoglobin level was less than $10 \mathrm{~g} / \mathrm{dl}$. Following adequate resuscitation, the patient was taken to theatre and had laparotomy through a transverse infra-umbilical incision under general anesthesia with endotracheal intubation. The bowel perforation was identified and the surgeon decides if to close the perforation primarily, do resection and anastomosis or an enterostomy created. The peritoneal cavity was mopped dry and abdomen closed in layers.

Post-operatively, graded oral intake was commenced when bowel function returned. The surgical site was examined on the $4^{\text {th }}$ day and patient discharged on the $5^{\text {th }}$ day if there are no problems. Patients who have had laparotomy for the same pathology at a peripheral hospital before referral to ESUTH for reoperation and patients with incomplete case records were excluded from this study. The ESUTH serves the whole of Enugu State, which according to the 2016 estimates of the National Population Commission and Nigerian National Bureau of Statistics, has a population of about 4 million people and a population density of $616.0 / \mathrm{km}^{2}$. The hospital also receives referrals from its neighboring states. Information was extracted from the case notes, operation notes, operation register, and admission-discharge records. The information extracted included the age, gender, duration of symptoms before presentation, time interval between presentation and surgery, operative procedure performed, post-operative complications, duration of hospital stay and outcome of treatment. The period of follow up was for 12 months. Ethical approval was obtained from the ethics and research committee of ESUTH. Statistical Package for Social Science (SPSS) version 21, manufactured by IBM Cooperation Chicago, Illonois, was used for data entry and analysis. Data were expressed as percentages, median, mean, and range.

\section{RESULTS}

\section{Patients' demographics}

Two hundred and nine children had laparotomy for TIP during the study period but only 200 cases had complete case records and were assessed. This accounted for $66.2 \%$ of the TIP operated on during the period of the study in our institution; 102 adults had laparotomy for TIP during the same period. Out of 560 pediatric surgeries performed during the study period, surgery for TIP accounted for $35.7 \%$. There were $150(75 \%)$ males and $50(25 \%)$ females which correspond to a male female ratio of $3: 1$. The ages of the patients ranged from 6 to 15 years with a median of 11 years. None of the patients was less than 5 years of age. The median duration of symptoms prior to presentation to the hospital was 6 days $(3-8)$. Twenty patients $(10 \%)$ presented within 24 hours of onset of symptoms while forty patients (20\%) presented between 24 and 72 hours. One hundred and forty patients (70\%) presented after 72 hours of onset of their symptoms. The median duration from presentation to surgery was 2 days (range: 1 - 5). The mean duration of hospital stay was 13.1 days (range 9-23), as shown in Table 1.

\section{Clinical presentation of the patients}

The patients presented with a constellation of symptoms which included abdominal pain, fever, abdominal swelling, diarrhea, vomiting and constipation. No sequence of symptoms is pathognomonic of typhoid intestinal perforation. Fever and abdominal pain were present in all the patients. Vomiting and abdominal distension were present in 183 patients (91.5\%). Diarrhea was documented in 66 patients (33\%) whereas 33 patients $(16.5 \%)$ had constipation in addition to fever and abdominal pain. The classic triad of fever, abdominal pain and abdominal distension were present in 185 patients (92.5\%).

Table 1. Demographic characteristics of the patients

\begin{tabular}{ll}
\hline Variables & \\
\hline Gender & $150(75 \%)$ \\
Male & $50(25 \%)$ \\
Female & 11 years (6-15) \\
Median age of the patients & 6 days $(4-12)$ \\
$\begin{array}{l}\text { Median duration of symptoms prior to } \\
\text { presentation }\end{array}$ & $4(9.1 \%)$ \\
$\begin{array}{l}\text { Presented within } 24 \text { hours } \\
\text { Presented between } 24 \text { and } 72 \text { hours }\end{array}$ & $8(18.2)$ \\
Presented after 72 hours & $32(72.7 \%)$ \\
$\begin{array}{l}\text { Median duration from presentation to } \\
\text { surgery }\end{array}$ & 2 days $(1-4)$ \\
The mean duration of hospital stay & 13.1 days $(9-23)$ \\
\hline
\end{tabular}


Table 2. Operative procedure performed

\begin{tabular}{ll}
\hline Operation performed & Number (\%) \\
\hline Primary closure & $170(85)$ \\
Ileostomy & $30(15)$ \\
Indication for relaparotomy & \\
Intestinal leak & $9(4.5)$ \\
Intra-peritoneal abscess & $9(4.5)$ \\
\hline
\end{tabular}

Investigation results

Imaging

All the patients had abdominopelvic ultrasound and presence of echo rich peritoneal fluid was seen in 178 patients (89\%). Plain $\mathrm{x}$ ray showed air under the diaphragm in 120 patients $(60 \%)$ and air-fluid level was seen in 181 patients (90.5\%).

\section{Laboratory}

Ninety percent (180 patients) had hemoglobin level of less than 10 gram per deciliter and serum electrolyte showed hypokalemia (potassium $<2.5$ millimoles per liter) in $70 \%$ of the patients ( $\mathrm{n}=140)$. Widal test was positive in all the patients.

\section{Operative findings}

Seventy percent of the patients $(n=140)$ had a single intestinal perforation whereas 30 percent of the patients $(n=60)$ had more than one perforation. All the perforations were in the ileum and the most proximal perforation was 45 centimeters to theileocecal valve. Each perforation was round/ovoid in shape measuring 1-2 centimeters at the antimesenteric border of the ileum. This operative finding is in line with typhoid intestinal perforation due to aggregation of lymphoid tissues (Peyer's patches) in the terminal ileum. The bacteria, Salmonella, commonly cause hyperplasia of the Peyer's patches. Necrosis and ulceration of Peyer's patches leads to intestinal perforation. No perforation was seen in the colon.

\section{Operative procedure performed}

The edges of the perforation were excised and closed primarily in 170 patients (85\%) whereas 30 patients (15\%) received an ileostomy after thorough cleaning of the peritoneal cavity. The excised edges of the perforations were sent for histopathological examination. Histology showed cryptic changes with evidence of acute and chronic inflammation on the Peyer's patches which supports the fact that the intestinal perforation was due to typhoid. Segmental bowel resection and right hemicolectomy were not performed in any of the patients. Eigh- teen (9\%) patients underwent relaparotomy; nine (4.5\%) patients for an intestinal leak and nine (4.5\%) for residual intra-peritoneal abscess, Table 2.

\section{Post-operative complications}

Ninety patients $(45 \%)$ had no complications and their wounds healed by primary intension. Thirty patients (15\%) has surgical site infection evidenced by purulent effluent from the wound and culture result that yielded a significant bacterial growth. Other complications are depicted in Figure 1.

\section{Treatment outcome}

One hundred and eighty three patients (91.5\%) of the patients did well and were discharged home. Mortality was recorded in 17 patients (8.5\%).

\section{DISCUSSION}

TIP is one of the most common surgical complications of typhoid fever and is associated with significant morbidity and mortality especially in developing countries [11, 12]. Typhoid causes ulceration of the Peyer's patches at the terminal ileum resulting in bowel perforation. This leads to leakage of intestinal contents leading to superinfection of the peritoneal cavity by the gut flora culminating in peritonitis ${ }^{[13]}$. Despite some measures such as public enlightenment and education, intestinal perforation by salmonellosis remains one of the most common emergency surgeries performed in children in our institution ${ }^{[14]}$.

In the present study, more laparotomies for TIP were performed in children than in adults. This finding was in accordance with the reports of other authors ${ }^{[10,15]}$. However, there is a report of typhoid perforation occurring in $20 \%$ of adults and $10.3 \%$ of children ${ }^{[16]}$. The predominant age group affected by typhoid perforation varies from place to place and with the economic status ${ }^{[10]}$. The male dominance recorded in the present study

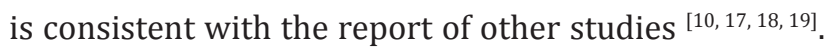
However, Olori et al. and Edino et al. recorded female predominance ${ }^{[20,21]}$. Khan tried to explain male dominance in typhoid perforation. He stated that the cytokine response to an intestinal infection in males is predominantly proinflammatory due to differences in sex hormones ${ }^{[22]}$. There are wide variations in the median ages of patients who have typhoid intestinal perforation. The median age of 11 years registered in the present study is the average of other studies ${ }^{[10,17,18]}$. None of the patients was less than 5 years of age. This finding is in agreement of the report of Usang et al ${ }^{[23]}$. However, there are re- 


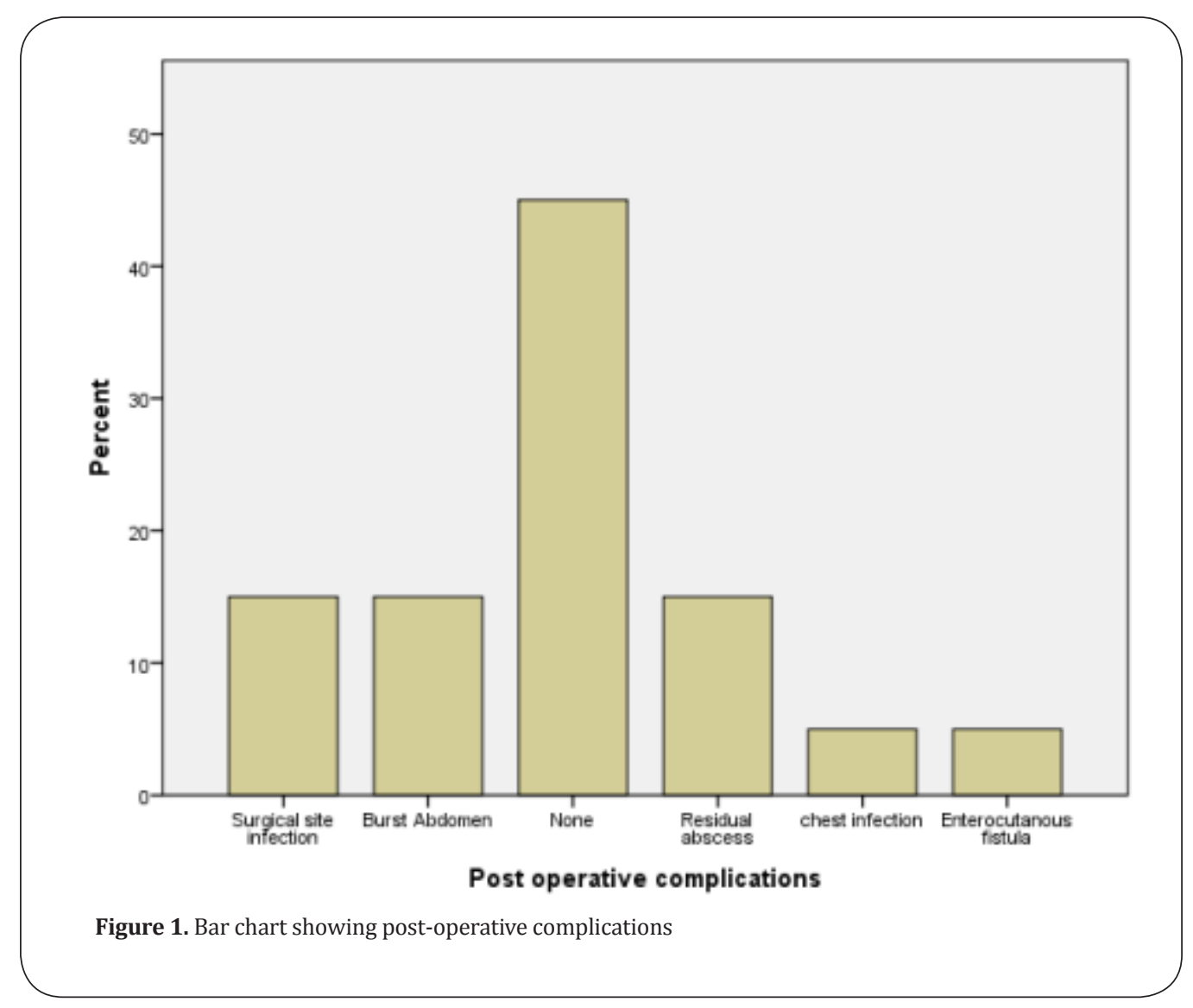

ports of typhoid intestinal perforation in children who are less than 5 years of age ${ }^{[16,24]}$. Typhoid intestinal perforation in under-fives has atypical presentation and is associated with high mortality ${ }^{[24]}$. Late presentation of our patients is manifested in the 6 day median period prior to presentation. This is consistent with the report of other studies emanating from developing countries $[16,18,19]$. This delayed presentation may be attributable to poverty and ignorance that is prevalent in developing countries.

The classic triad of fever, abdominal pain and abdominal distension were not present in all our patients. However, a significant proportion of our patients had the triad. This is in agreement with the report of other series ${ }^{[8,15,}$ ${ }^{23]}$. Fever and abdominal pain were consistent presentation in most studies on typhoid intestinal perforation $[8,16,23]$. Other symptoms such as diarrhea and vomiting could be present depending on the time of presentation ${ }^{[23]}$. In the present study, plain radiograph showed air under the diaphragm in a significant number of patients. This finding is in line with the reports of other studies on typhoid intestinal perforation ${ }^{[25,26]}$. Abdominal ultrasound for typhoid perforation usually shows free peritoneal fluid that is echo rich. This was recorded in $89 \%$ of our patients similar to the reports of other workers
[25, 26]. The presence or absence of free peritoneal fluid may depend on the number of perforation and time of presentation to the hospital. Experience and expertise of the sonographer may also count. Anemia and hypokalemia are frequent findings in typhoid perforation ${ }^{[23]}$. Ninety percent and $70 \%$ of our patients were anemic and hypokalemic respectively. This finding is consistent with the report of other researchers ${ }^{[17,19,23,26]}$. Vomiting, diarrhea, bleeding and leakage of intestinal contents into the peritoneal cavity may explain the anemia and hypokalemia. The poor nutritional status of the patients prior to the intestinal perforation may contribute to the anemia. Majority of our patients had a single ileal perforation. Several other studies also reported single perforation as the most common form of typhoid perforation ${ }^{[19,20,25,26]}$. However, the number of perforations does not affect the severity of symptoms ${ }^{[27]}$. Neither does the number of perforations associated with adverse outcome ${ }^{[18]}$. Although there were no colonic perforations in the present study, some authors have reported colonic involvement ${ }^{[3,28]}$. Some researchers have postulated that colonic involvement in typhoid perforation is due to direct bacterial invasion while ileal perforation is due to enterotoxin produced from parasitized macrophages that caused hyperplasia, necrosis and ulceration of the 
Peyer's patches ${ }^{[26,29]}$.

There are options of treatment of typhoid perforation. Excision of the edges of the perforation and primary closure in 2 layers were the most performed surgical procedure in the index study and in several other studies too ${ }^{[15,17,18,19,25,26]}$. A study conducted in northern Nigeria reported segmental bowel resection and anastomosis as the best option for treatment of TIP ${ }^{[16]}$. However, in a background of multiple perforations and gross peritoneal contamination, creation of ileostomy may be lifesaving ${ }^{[3]}$. The experience of the surgeon and patients' factors may determine the best option of treatment for a particular patient.

Surgical site infection, burst abdomen and residual abscess were most common complications observed in the present study. Other published series also reported similar complications ${ }^{[10,17,19]}$. Chest infection in the post-operative period also contributes to post-operative morbidity ${ }^{[16,23]}$. In the present study, mortality recorded is similar to a report from Kano, northern Nigeria ${ }^{[18]}$. There were wide variations in mortality rates of patients that have typhoid intestinal perforation; the reported mortality rate lies in the range of $5 \%$ to $80 \%{ }^{[27]}$. Outcome of treatment of patients who have typhoid perforation depends on multiple factors; it could be patient related or surgeon related ${ }^{[27]}$.

\section{CONCLUSION}

Typhoid fever, caused by gram-negative flagellated facultative anaerobic bacteria (Salmonella), may be complicated by intestinal perforation. Lack of potable drinking water, poor hygiene and improper waste disposal has made typhoid intestinal perforation a persistent problem in developing countries.

\section{DECLARATION}

Authors' contribution

The author contributed solely to this article.

Availability of data and materials

Data is available with the author and can be provided on request.

\section{Conflict of interest}

The author declares that there is conflict of interest.

\section{Ethical approval}

Ethical approval was obtained from the hospital ethics committee.

\section{REFERENCES}

1. Ochiai, R. L., Acosta, C. J., Danovaro-Holliday, M., Baiqing, D., Bhattacharya, S. K., Agtini, M. D., ... \& Wain, J. (2008). A study of typhoid fever in five Asian countries: disease burden and implications for controls. Bulletin of the world health organization, 86, 260-268.

2. Badoe, E. A., Archampong, E. Q., \& Jaja, M. O. A. (Eds.). (1994). Principles and Practice of Surgery, Including Pathology in the Tropics. University of Ghana Medical School.

3. Sharma, A. K., Sharma, R. K., Sharma, S. K., Sharma, A., \& Soni, D. (2013). Typhoid intestinal perforation: 24 perforations in one patient. Annals of medical and health sciences research, 3(1a), 41-43.

4. Parry, C. M., Hien, T. T., Dougan, G., White, N. J., \& Farrar, J. J. (2002). Typhoid fever. The New England journal of medicine, 347(22), 1770-1782.

5. Bitar, R., \& Tarpley, J. (1985). Intestinal perforation in typhoid fever: a historical and state-of-the-art review. Reviews of infectious diseases, 7(2), 257-271.

6. Worku, B. (2000). Typhoid fever in an Ethiopian children's hospital: 1984-1995. Ethiopian Journal of Health Development, 14(3).

7. Chiu,C.H., Tsai,J.R.,Ou,J.T., \& Lin, T.Y.(2000). Typhoid fever in children: a fourteen-year experience. Acta paediatrica Taiwanica $=$ Taiwan er ke yi xue hui za zhi, 41(1), 28-32.

8. Ekenze, S. O., \& Okoro, P. E. (2008). Typhoid ileal perforation: Analysis of morbidity and mortalityin 89 children. Nigerian journal of clinical practice, 11(1), 5963.

9. Mogasale, V., Desai, S. N., Mogasale, V. V., Park, J. K., Ochiai, R. L., \& Wierzba, T. F. (2014). Case fatality rate and length of hospital stay among patients with typhoid intestinal perforation in developing countries: a systematic literature review. PLoS One, 9(4).

10. Osifo, O. D., \& Ogiemwonyi, S. O. (2010). Typhoid ileal perforation in children in Benin city. African Journal of Paediatric Surgery, 7(2), 96.

11. Keenan, J. P., \& Hadley, G. P. (1984). The surgical management of typhoid perforation in children. British journal of surgery, 71(12), 928-929.

12. Ajao, O. G. (1982). Typhoid perforation: factors affecting mortality and morbidity. International surgery, 67(4), 317-319.

13. Ahmad, T., Khan, M. I., Hussain, N., Siddiqui, E., \& Islam, Z. U. (2009). Perforation operation interval as a prognostic factor in typhoid ileal perforation. J Surg Pakistan (International), 14, 11-14.

14. Emeka, C. K., Chukwuebuka, N. O., Kingsley, N. I., Arinola, O. O., Okwuchukwu, E.S., \& Chikaodili, E. T. (2019). Paediatric Abdominal Surgical Emergencies in Enugu, South East Nigeria: Any Change in Pattern and Outcome. European Journal of Clinical and Biomedical Sciences, 5(2), 39-42. 
15. Grema, B. A., Aliyu, I., Michael, G. C., Musa, A., Fikin, A. G., Abubakar, B. M., \& Olusegun, S. (2018). Typhoid ileal perforation in a semi-urban tertiary health institution in north-eastern Nigeria. South African Family Practice, 60(5), 168-173.

16. Ameh, E. A. (1999). Typhoid ileal perforation in children: a scourge in developing countries. Annals of tropical paediatrics, 19(3), 267-272.

17. Uba, A. F., Chirdan, L. B., Ituen, A. M., \& Mohammed, A. M. (2007). Typhoid intestinal perforation in children: a continuing scourge in a developing country. Pediatric surgery international, 23(1), 33-39.

18. Sheshe, A. A., Anyanwu, L. J. C., Mohammad, A. M., Muhammad, A. B., \& Obaro, S. K. (2018). Typhoid intestinal perforation: Analysis of the outcome of surgical treatment in Kano, Nigeria. Archives of Medicine and Health Sciences, 6(1), 59.

19. Nuhu, A., Gali, B., \& Dawha, S. (2009). Postoperative complications of typhoid ileal perforation in children in Azare, Nigeria. Internet J Surg, 21.

20. Edino, S. T., Yakubu, A. A., Mohammed, A. Z., \& Abubakar, I. S. (2007). Prognostic factors in typhoid ileal perforation: a prospective study of 53 cases. Journal of the national medical association, 99(9), 1042.

21. Olori, S., \& Ukpoju, E. (2019). Typhoid ile al pe rforation in childr en: does clinical diagnosis alone jus tify laparotomy?. South Sudan Medical Journal, 12(1), 9-11.

22. Khan, M. (2012). A plausible explanation for male dominance in typhoid ileal perforation. Clinical and experimental gastroenterology, 5, 213.

23. Usang, U. E., Inyang, A. W., Nwachukwku, I. E., \& Emehute, J. D. C. (2017). Typhoid perforation in children: an unrelenting plague in developing countries. The Journal of Infection in Developing Countries, 11(10), 747-752.

24. Ekenze, S. O., \& Ikefuna, A. N. (2008). Typhoid intestinal perforation under 5 years of age. Annals of tropical paediatrics, 28(1), 53-58.

25. Ugochukwu, A. I., Amu, O. C., \& Nzegwu, M. A. (2013). Ileal perforation due to typhoid fever-review of operative management and outcome in an urban centre in Nigeria. International Journal of Surgery, 11(3), 218-222.

26. Chalya, P. L., Mabula, J. B., Koy, M., Kataraihya, J. B., Jaka, H., Mshana, S. E., ... \& Gilyoma, J. M. (2012). Typhoid intestinal perforations at a University teaching hospital in Northwestern Tanzania: A surgical experience of 104 cases in a resource-limited setting. World journal of emergency surgery, 7(1), 4.

27. Contini, S. (2017). Typhoid intestinal perforation in developing countries: Still unavoidable deaths?. World journal of gastroenterology, 23(11), 1925

28. Chang, Y. T., Lin, J. Y., \& Huang, Y. S. (2006). Typhoid colonic perforation in childhood: a ten-year experience. World journal of surgery, 30(2), 242-247.

29. Elesha, S. 0. (1994). Pathology and pathogenesis of typhoid fever. Nig P Med J, 1, 38. 